\title{
A análise da eficiência no setor bancário: \\ Modelo de fronteira estocástica com dados em painel para a banca portuguesa
}

\begin{abstract}
Palavras-chave
indústria bancária, funções custo, X-ineficiência, fronteira de custo estocástica, modelos com dados em painel.
\end{abstract}

Classificação JEL C33, D2, G21

\section{Key-words \\ banking industry, cost function, $X$-inefficiency, stochastic cost frontier, panel data analysis.}

JEL Classification C33, D2, G21

\section{Resumo}

O objetivo deste estudo é a análise da eficiência produtiva sobre os custos bancários, tendo por base a banca portuguesa. Nesta investigação adotou-se a abordagem de intermediação na conceItualização da empresa bancária. Incluem-se na análise da eficiência os custos financeiros além dos operacionais. A especificação custo adotada é a forma funcional Fourier devido ao caráter multiproduto da empresa bancária.

A amostra corresponde à quase totalidade dos bancos portugueses com dados não consolidados em painel. $\mathrm{O}$ estudo da ineficiência é elaborado recorrendo ao modelo estocástico da curva fronteira. A taxa de ineficiência média estimada é de cerca de 4\%. Em síntese, o processo de concentração, ainda inacabado, do sector bancário português, parece justificar-se pela possibilidade de diminuição da ineficiência-X.
Francisco Vitorino da Silva Martins Universidade do Porto, Faculdade de Economia

Maria Clara Dias Pinto Ribeiro CECEJ - Centro de Estudos em Ciências Empresariais e Jurídicas, Instituto Politécnico do Porto, Instituto Superior de Contabilidade e Administração do Porto

\section{Abstract}

This study addresses the productive efficiency regarding the banking costs, stressing its focus on the Portuguese banking market. This study follows the intermediation option concerning the conceptualization of the banking firm. The financial and operating costs are both considered in the efficiency analysis. The costspecification used is the Fourier functional form because of the multiproduct nature of the banking firm. The sample covers nearly all the Portuguese banks with non-consolidated panel data. The study of inefficiency is elaborated through the stochastic frontier model. The inefficiency rate is estimated on about $4 \%$. Summing up, the yet unfinished concentration process of the Portuguese Banking Industry seems to be justified by the possibility of shrinkage of the X-inefficiency. 


\section{Introdução}

Os primeiros estudos de economia bancária remontam aos anos cinquentas e tiveram como gênese o processo de concentração ocorrido no setor bancário norte-americano nessa década. Cumulativamente, as grandes transformaçóes ocorridas no setor bancário europeu serviram de base a numerosos estudos empíricos a partir, sobretudo, dos anos setentas. Atualmente, parece haver um interesse acrescido pela análise de setores bancários, além dos do eixo Estados Unidos da América/Europa Ocidental que se debruçam sobre bases de dados que vão desde os países emergentes do Sudeste Asiático aos países do Leste Europeu, da América Latina ou da Austrália. Muitos dos trabalhos incidem sobre a mensuração da eficiência produtiva e das economias de escala e de gama, enquadradas por variadas especificaçóes de funções de produção, custo ou lucro e recorrendo a diferentes processos de estimação.

A análise do setor bancário passa pela definição de empresa bancária (consoante a adoção da abordagem produção ou da abordagem intermediaçāo), pelo cômputo da ineficiência produtiva, pelas especificações funcionais de funçóes produção, custo ou lucro e pelos diversos processos de estimação.

Com o propósito de analisar a eficácia de funcionamento das empresas, Lei- benstein (1966) introduziu o importante conceito em Economia Industrial - o conceito de ineficiência-X ou ineficiência custo. Esse conceito foi adotado pela economia bancária, sugerindo, alguns autores, que a ineficiência-X está associada a mais de $20 \%$ da totalidade dos custos. Para a medida da ineficiência-X, recorre-se a diferentes métodos que pretendem medir a distância entre a fronteira de produção eficiente e a situação específica da empresa. Com esse propósito, têm sido desenvolvidos pela literatura bancária alguns métodos de aproximaçóes paramétricas e não paramétricas para a mensuração da ineficiência produtiva. Os resultados obtidos apresentam-se diversos, consoante o método e a própria especificação de custo adotados.

O objetivo do presente trabalho é o estudo da eficiência produtiva, tendo por base o mercado bancário português. $\mathrm{O}$ estudo da eficiência faz-se recorrendo ao modelo de fronteira estocástica (SFA), com a análise da especificação Fourier da função custo, tendo como amostra 22 bancos que operam em Portugal, em 31 de dezembro, entre 1995 e 2001. O modelo proposto inclui quatro inputs para melhor interpretar os recursos produtivos que mais contribuem para a eficiência produtiva e para detectar eventuais falhas na gestão deficiente desses recursos. 
O processo de estimação tem em atenção os dados em painel. Esta análise surge como uma das mais inovadoras e promissoras linhas de desenvolvimento econométrico (tanto ao nível das técnicas de estimação como ao dos resultados teóricos). O recurso a dados em painel permite considerar a existência de efeitos não observáveis, específicos a cada uma das instituições de crédito, ultrapassando, em parte, o problema da existência de variáveis omitidas (o que provoca enviesamento no processo de estimação).

Este trabalho é dividido em três pontos. No primeiro, enquadra-se a empresa bancária no âmbito do corpo da teoria da empresa, em particular relevando os conceitos aplicáveis a empresas multiproduto; em seguida, apresentam-se os debates teóricos recentes na economia bancária, nomeadamente quanto à definição de empresa bancária e acerca das formas funcionais da função custo bancária. É objeto do segundo ponto a discussão dos diferentes conceitos de eficiência e a sua mensuração, por via de modelos estocásticos. $\mathrm{O}$ terceiro ponto é dedicado à estimação da eficiência produtiva, recorrendo ao modelo de fronteira estocástica, com base numa amostra de dados em painel de 22 instituiçóes de crédito que operam em Portugal, tendo em conta o período de 1995 a 2001 (dados não consolidados, de 31 de dezembro).
A completar o presente trabalho, são apresentadas as principais ideias e as conclusóes fundamentais que podem ser extraídas sobre a banca nacional com base nas aplicações empíricas.

\section{2_Os debates recentes sobre a empresa bancária}

Atualmente, existem dois debates fundamentais, e ainda não resolvidos, na literatura sobre a empresa bancária. $\mathrm{O}$ primeiro quanto à definição de empresa bancária e o segundo acerca da especificação da função custo (ou produção), comportando implicações quanto à medida da eficiência.

\section{1_A definição de empresa bancária}

A especificação de uma medida adequada do output de uma empresa bancária, ou seja, o que é que um banco produz e como se pode medir essa produção, é um dos debates teóricos não resolvidos na literatura de economia bancária. Ao contrário de uma empresa industrial, o output de um banco não pode ser medido por meio de quantidades físicas; a acrescer a essa dificuldade, uma empresa bancária caracteriza-se por ser multiproduto.

A estimação de funçóes de produção e de custos aplicadas à atividade bancária não foi objeto de estudos senão a partir de finais dos anos sessentas. Uma das razóes 
explicativas dessa tardia aplicação decorre da dificuldade de medição das produçóes bancárias decorrente da impossibilidade da sua mensuração em termos físicos.

O processo de produção bancária caracteriza-se pela captação de uma série de informação, pelo seu tratamento e pela disponibilização de nova informação sob a forma de serviços e de produtos financeiros.

A medida da produção bancária tem sido objeto de numerosas leituras, divergindo com base em questões teóricas e outras com suporte em problemas de mera operacionalidade - informação disponível e processos de estimação. ${ }^{1}$ Com efeito, a definição de produção bancária e a sua mensuração teve, nessas últimas três décadas, numerosas opçóes como o total do ativo, os depósitos totais, os depósitos à ordem, as aplicaçóes creditícias a particulares e empresas e os créditos interbancários, as operaçóes em titulos, a soma da margem financei$r a$ (definida como a diferença entre juros $e$ proveitos equiparados e juros e custos equiparados) e outros resultados correntes (diferença entre proveitos por natureza e custos por natureza), o número de contas de aplicaçooes creditícias e de depósitos. ${ }^{2}$

Toda essa problemática da definição da produção bancária tem como suporte teórico a definiçấo econômica de empresa bancária que trouxe à literatura duas aproximações: a da produção e a da inter- mediação (Humphrey, 1985). Segundo a abordagem produção, a empresa bancária é uma empresa de serviços caracterizada por captar recursos (depósitos à ordem, depósitos a prazo) com vista à sua aplicação (créditos, aplicaçóes em títulos, participaçóes de capital), ou seja, as empresas bancárias são produtoras de serviços associadas a créditos e depósitos, sendo os fatores de produção o trabalho e o capital. Os custos totais considerados nas análises empíricas excluem, logicamente, os encargos financeiros, comportando somente os custos operatórios ou de produção. Segundo a ótica da intermediação, desenvolvida a partir de finais dos anos setentas (Sealey; Lindley, 1977), a empresa bancária é vista como simples intermediária nos mercados financeiros, ou seja, o processo produtivo exige a recolha ou o empréstimo de fundos que serão, em seguida, objeto de aplicação ou empréstimo. Nessa ótica, além do trabalho e do capital, os depósitos são considerados como inputs. Consistentes com essa abordagem, nos custos totais, são incluídos os custos operacionais ou de produção e os custos financeiros.

Sendo as duas abordagens originais - produção e intermediação - conceitualmente muito diferentes, os resultados empíricos quanto ao cômputo da eficiência parecem arrastar grandes alte-
${ }^{1}$ D'Oliveira (1984,

p. 10-11, 27-31).

2 Para uma revisão da literatura em torno da mensuraçáo do output bancário, ver Molyneux, Altunbas e Gardener (1996, p. 152-156). 
${ }^{3}$ Ao contrário do que sucede no cômputo de economias de escala e de gama, em que as diferentes concepçóes não parecem interferir grandemente nos resultados.

${ }^{4}$ Para mais desenvolvimentos, ver, por exemplo, Fare e Primont (1997).

${ }^{5}$ Jehle (1991, p. 238).

${ }^{6}$ Referido por Molyneux, Altunbas e Gardener (1996, p. 150). rações nos resultados (Barros; Soares de Pinho, 1995, p. 41). ${ }^{3}$

Berger, Hanwecke Humphrey (1987, p. 511) sustentam que a escolha apropriada entre as duas aproximaçóes depende da questão avançada, mas a aproximação intermediação é preferível dado ser mais inclusiva da totalidade dos custos bancários. Parece-nos, assim, a abordagem intermediação mais apropriada para a caracterização da empresa bancária, em razão do importante peso da atividade interbancária e dos custos financeiros na totalidade dos custos. A justificar tal escolha temos ainda uma razão de ordem empírica: no caso português, a informação quanto ao número de contas de crédito e de depósitos, nas diferentes empresas bancárias, não se encontra disponível, inviabilizando, à partida, a aplicação da abordagem produção (Mendes, 1994).

\section{2_A especificação da função custo}

A função custo pode ser estabelecida a partir da função de produçáo sob certas condições, permitindo uma abordagem alternativa, via custos, das condiçóes tecnológicas do processo produtivo.

A aplicação da teoria da dualidade, desenvolvida por R. W. Shephard, e, em particular, o lema de Shephard ${ }^{4}$ permite concluir que, sob determinadas condiçóes de regularidade, as funçóes custo e de pro- dução são duais uma em relação à outra. Essa conclusão é particularmente importante na execução de estudos empíricos:

\begin{abstract}
Applied researchers need no more longer begin their study of the firm with detailed knowledge of the technology and with access to relatively obscure data. Instead, they can concentrate on devising and estimating flexible functions of observable market prices and output and be assured that they are carrying along all economically relevant aspects of the underlying technology. ${ }^{5}$
\end{abstract}

Diewert (1992) demonstra que o recurso a funçóes custo em detrimento das funções produção tem a vantagem de simplificar o processo de estimação, além de que os parâmetros da função custo podem ser estimados de forma precisa. Binswanger $(1974)^{6}$ mostrou que as funçóes custo são homogêneas em relação aos preços, não sendo necessária a existência da homogeneidade de grau um na função de produção para o processo de estimação; por outro lado, o problema da existência de elevada multicoliniaridade entre as variáveis inputs que ocorre no processo de estimação das funções de produção não ocorre na estimação das funçóes custo (não existe, regra geral, alta multicolinearidade entre os preços dos inputs). 
Finalmente, da teoria da produçáo, decorrendo da dualidade entre produção e custos, a função custo apresenta as seguintes propriedades: 7 positividade (a função custo é positiva para valores positivos dos preços dos inputs e para níveis positivos de output); homogeneidade (a função custo é homogênea de grau um em relação aos preços dos inputs); monotonicidade (a função custo é crescente com os preços dos inputs e com o nível da produção); e concavidade (a função custo é côncava).

Mas quais são as razóes, a priori, que poderão fazer com que se escolha uma forma para uma função custo multiproduto (ou uma função de produção) em detrimento de outra? Por outras palavras, quais as propriedades desejáveis para uma função custo multiproduto?

Seguindo Lau (1986), podemos agrupar essas propriedades em cinco grupos: consistência teórica, domínio de aplicação, flexibilidade, facilidade de tratamento informático e conformidade com os dados empíricos. A consistência teórica requer que a forma funcional para a função custo cumpra as propriedades apontadas por Jorgenson (1986), pelo menos localmente. O critério do domínio de aplicação refere-se ao conjunto de valores das variáveis independentes para o qual são cumpridas as condiçóes decorrentes da consistência teórica; da mesma forma que anteriormente, o domínio de aplicação pode não corresponder a todas as escolhas dos parâmetros, mas apenas para um pequeno conjunto de preços dos inputs estar assegurada a consistência teórica. A flexibilidade da forma funcional da função custo refere-se à não exigência de quaisquer restriçóes nos valores na primeira e segunda derivadas parciais, ou seja, permite que os dados empíricos forneçam informação acerca do comportamento dos parâmetros.

A facilidade do recurso a tratamento informático das formas funcionais da funçâo custo implica que: os parâmetros possam ser inferidos claramente dos dados (conforme Diewert, 1992); o número de parâmetros a estimar deve ser o menor possível (em muitos casos o número de observaçóes é relativamente reduzido e da necessidade da estimação de um número elevado de parâmetros pode decorrer perda de graus de liberdade excessiva). A conformidade com os dados empíricos conhecidos tem de ser assegurada pela forma funcional da função custo escolhida.

A forma da Translog híbrida, já testada empiricamente, parece cumprir razoavelmente os cinco grupos de critérios apontados. No entanto, alguns estudos (Altunbas; Liu; Molyneux; Seth, 2000 e Humphrey; Vale, 2003) defendem a pouca flexi-
${ }^{7}$ Seguindo Jorgenson (1986, p. 1841-1915). 
bilidade da forma Translog, optando por formas mais flexíveis (no caso a Fourier - Altunbas; Liu; Molyneux; Seth, 2000 ou a Fourier e a Spline - Humphrey; Vale, 2003).

A função Fourier é uma forma funcional mista ou semiparamétrica, construída a partir de uma Translog completa e incluindo os termos trigonométricos de primeira, segunda e terceira ordens.

$$
\begin{aligned}
& \ln C T=\alpha_{0}+\sum_{i=1}^{m} \alpha_{i} \ln Q_{i}+\sum_{j=1}^{n} \beta_{j} \ln W_{j}+\frac{1}{2} \sum_{i=1}^{m} \sum_{j=1}^{m} \delta_{i j} \ln Q_{i} \ln Q_{j}+ \\
& +\frac{1}{2} \sum_{i=1}^{n} \sum_{j=1}^{n} \gamma_{i j} \ln W_{i} \ln W_{j}+\sum_{i=1}^{m} \sum_{j=1}^{n} \rho_{i j} \ln Q_{i} \ln W_{j}+\sum_{i=1}^{m}\left(\sigma_{i} \cos z_{i}+\theta_{i} \sin z_{i}\right)+ \\
& +\sum_{i=1}^{m} \sum_{j=1}^{m}\left[\sigma_{i j} \cos \left(z_{i}+z_{j}\right)+\theta_{i j} \sin \left(z_{i}+z_{j}\right)\right]+ \\
& +\sum_{i=1}^{m} \sum_{j=1}^{m} \sum_{k=j}^{m}\left[\sigma_{i j k} \cos \left(z_{i}+z_{j}+z_{k}\right)+\theta_{i j k} \sin \left(z_{i}+z_{j}+z_{k}\right)\right]
\end{aligned}
$$

em que CT representa o custo total, $Q_{i}$ o nível do output i, $W_{j}$ o preço do input je $z_{i}$ os valores “ajustados" de $\ln Q_{i}$ de forma a variarem no intervalo $[0 ; 2 \pi] .{ }^{8} \mathrm{O}$ cálculo de $z_{i}$ é dado por $z_{i}=0,2 \pi-\mu \times a+\mu \times \ln Q_{i}$, onde $[a ; b]$ é o intervalo de variação de $\ln Q_{i}$ e $\mu=\frac{0,9 \times 2 \pi-0,1 \times 2 \pi}{b-a}$.

Carbot, Gardener e Williams (2003), Altunbas, Liu, Molyneux e Seth (2000) e Altunbas, Gardener, Molyneux e Moore (2001) - seguindo Mitchell e Onvural (1996) - adotaram, para a especificação da Fourier, os termos trigonométricos de primeira e de segunda ordens. Os autores baseiam-se nos trabalhos de Gallant (1981, 1982), segundo o qual se consegue obter boa aproximação da verdadeira função custo, recorrendo a um número limitado de termos trigonométricos. ${ }^{9}$ De fato, foi mostrado por Tolstov (1962) que uma combinação linear das funções seno e cosseno (séries Fourier) pode ajustar exatamente o comportamento de uma função multivariável (uma vez que as funçóes seno e cosseno são mutuamente ortogonais no intervalo $[0 ; 2 \pi])$.

No caso da forma funcional Fourier, como resultante da Translog, as equaçóes dos "shares" podem ser explicitadas, 
atendendo ao Lema de Shephard, de forma idêntica: ${ }^{10}$

$S_{i}=\beta_{i}+\sum_{j=1}^{n} \gamma_{i j} \ln W_{j}+\sum_{i=1}^{m} \rho_{i j} \ln Q i$

Da mesma forma, sendo n o número de fatores de produção e dado que a soma dos "shares" tem de ser um, apenas n-1 equações podem ser estatisticamente independentes.

Uma vez que o teorema da dualidade exige que a função custo seja linearmente homogênea em relação aos preços dos inputs, os parâmetros têm de obedecer às seguintes restriçóes:

$$
\left\{\begin{array}{c}
\sum_{j=1}^{n} \beta_{j}=1 \\
\sum_{j=1}^{n} \gamma_{i j}=0, \quad \forall i \\
\sum_{i=1}^{n} \rho_{i j}=0, \quad \forall j
\end{array}\right.
$$

Igualmente, como na função Trans$\log$, as condiçóes de simetria dos parâmetros têm de estar asseguradas na Fourier $\left(\delta_{i j}=\delta_{j i}\right.$ para $\mathrm{i}>1, \mathrm{~m}>\mathrm{j}$ e $\gamma_{i j}=\gamma_{j i}$ para $\mathrm{i}>1, \mathrm{n}>\mathrm{j})$.

Berger, Leusner e Mingo (1997) defendem que a função tipo Fourier é uma aproximação preferível à função custo Translog, quando se estuda uma indústria multiproduto, como é o caso da bancária, uma vez que apresenta maior adaptação aos dados:

The Fourier-flexible form is a global approximation because the terms such $\cos z_{i}, \sin z_{i}, \cos 2 z_{i}, \sin 2 z_{i}$, are mutually orthogonal over the $[0 ; 2 \pi]$ interval, so that each additional term can make the approximating function closer to the true path of the data wherever it is most needed. ${ }^{11}$

De igual forma, Altunbas, Liu, Molyneux e Seth (2000) sustentam que a função Fourier tem propriedades matemáticas e estatísticas desejáveis, uma vez que é capaz de representar qualquer função com exatidão (mesmo truncada, a forma Fourier pode representar razoavelmente qualquer função). Ao contrário da forma funcional Translog, em que se assume que a função custo da indústria bancária tem, obrigatoriamente, a forma em U (do que, a não ser verdade, decorrerão erros na especificação), a formula- para esse procedimento. A aplicação de Dietsch e Lozano-Vivas (2000, p. 991), nota de rodapé 2 , vai em sentido contrário.

${ }^{11}$ Berger, Leusner e Mingo (1997, p. 147). 
ção Fourier permite que os dados determinem a forma da função custo. ${ }^{12}$

Um problema que se pode colocar com a aplicação da forma funcional tipo Fourier é a dificuldade na interpretação dos resultados, já que a existência de um número tão elevado de variáveis implica elevado grau de multicolinearidade.

\section{3_A estimação da eficiência no setor bancário}

$O$ conceito de eficiência-X foi introduzido por Leibenstein (1966) com o objetivo de analisar a eficácia do funcionamento no seio das empresas. A teoria da empresa tradicional (de origem neoclássica) pressupóe que cada unidade produtiva otimize o seu comportamento em relação quer

${ }_{12}$ No entanto, num estudo posterior, Altunbas e Chakravarty (2001, p. 239, nota de rodapé) apresentam algumas reservas à aplicabilidade crescente da Fourier nos estudos de economia bancária: The underlying critique [...] rest on the proposition that the mechanical approaches above are unlikely to provide economic insight into the banking system. A acrescer a essa suspeição, os autores chamam a atenção para o fato de o melhor ajustamento aos dados náo ser sinônimo de melhor capacidade de previsão (afirmação que é aplicável a qualquer processo de estimação).

${ }^{13}$ Berger, Hunter e Timme (1993, p. 222).

${ }^{14}$ Contrariando as conclusões de Berger, Hunter e Timme (1993), Altunbas, Liu, Molyneux e Seth (2000), para o caso específico do Japáo, obtiveram como resultado que as ineficiências ligadas à escala superavam a ineficiência - X. aos fatores de produção quer aos produtos; se o não fizer, o processo concorrencial fará com que as empresas menos eficientes sejam banidas do mercado. Leinbenstein afirma que a existência de ineficiência-X é a regra, ou seja, as empresas que operam segundo a minimização dos custos (sobre a curva fronteira) são exceções, constituindo as barreiras naturais à entrada ou processos regulamentares, as condiçóes permissivas da manutenção de empresas ineficientes no mercado. A consideração da existência de ineficiência custo constitui, portanto, um ponto de clivagem com a teoria neoclássica da empresa, em que se assume a eficiência dos produtores $(\mathrm{Ca}-$ nhoto, 1999, p. 29).

Diferenças de comportamento de gestão no controlo dos custos (ou de maximização dos rendimentos), imperfeiçôes do mercado ou imposiçóes regulamentares parecem, no seu conjunto, ser relativamente mais importantes para o estudo da eficiência do que a escolha da escala ou da gama. Berger, Hunter e Timme (1993), ${ }^{13}$ na sua revisão dos estudos sobre eficiência, afirmam que a ineficiência-X é responsável por mais de $20 \%$ da totalidade dos custos bancários, ao passo que as ineficiências ligadas à escala e à gama não ultrapassavam os $5 \%$ dos custos. ${ }^{14}$

O processo de estimação baseado nas formas funcionais já mencionadas 
não permite uma separação direta entre a ineficiência decorrente da escala e a ineficiência-X. Por outro lado, o maior problema para o cômputo da ineficiência é conseguir separar a efetiva ineficiência de outros fatores aleatórios que afetem o comportamento dos custos. ${ }^{15}$

Para tentar resolver tal dificuldade no cômputo da eficiência-X, foram utilizados na literatura bancária diferentes métodos, nomeadamente o método SFA (stochastic frontier approach), o método TFA (thick frontier approach), o método DFA (distribution - free approach) ou o método DEA (data envelopment analysis). ${ }^{16}$

Refere-se em seguida, em pormenor, o método estocástico que é usado, mais recorrentemente, nos estudos sobre a empresa bancária para a estimação das funções custo fronteira - SFA (stochastic frontier approach). O método SFA (stochastic frontier approach) avança que os custos observados num banco podem desviar-se da curva fronteira eficiente em razáo da ineficiência ou de flutuaçóes aleatórias; para separar essas duas parcelas, assume-se que o termo da ineficiência segue, habitualmente, uma distribuição assimétrica seminormal, e os erros aleatórios seguem uma distribuição simétrica normal (Aigner; Lovell; Schmidt, 1977). Pode ser aplicada a dados cross section ou a dados em painel.
Nessa aproximação, a função custo tem três parcelas: a primeira correspondente aos custos dos produtores mais eficientes, a segunda derivada da ineficiência-X (montante dos custos associados ao desperdício de recursos) e a terceira decorrente dos efeitos aleatórios não controlados pela empresa.

$$
C T=C T\left(Q_{i}, W_{i}, B_{i}\right)+u_{i}+v_{i}
$$

A $C T\left(Q_{i}, W_{i}, B_{i}\right)$ designa-se por curva custo fronteira ou fronteira estocástica -ou seja, são estimados os custos necessários para atingir determinado nível de produção sem desperdícios de recursos. A curva fronteira representa a relação entre custos, níveis de produçóes e preços dos fatores produtivos dos bancos relativamente mais eficientes, isto é, os de "melhor prática". Se $v_{i}=0$, o modelo da fronteira estocástica (SFA) coincide com o modelo da fronteira determinística.

A $u_{i}$ impóe-se que seja estritamente positivo, uma vez que a ineficiência aumenta os custos; habitualmente assume-se que $u_{i}$ segue uma distribuição seminormal. A $v_{i}$ associa-se uma distribuição normal de média zero e com desvio padráo de $\sigma_{v}$. Assume-se ainda que o erro da função custo, $\varepsilon$, é dado por $\varepsilon=u+v$, e $u$ e $v$ são variáveis aleatórias independentes.

\footnotetext{
${ }^{15} \mathrm{Na}$ nossa pesquisa, vamos trabalhar exclusivamente com funçóes custo, e não com funçóes lucro. Apesar das vantagens que o recurso a funçóes lucro pode trazer, em particular para a análise das fusóes e aquisiçóes (Berger, 1995), a especificação da função lucro exige que se conheçam os preços dos inputs e dos outputs.

${ }^{16}$ Para mais desenvolvimentos, ver Resti (2000).
} 
A sua função densidade de probabilidade será dada por:

$g(\varepsilon)=\frac{2}{\sigma} f\left(\frac{\varepsilon}{\sigma}\right)[1-F(\varepsilon \lambda / \sigma)]$

sendo $\sigma=\sqrt{\sigma_{u}^{2}+\sigma_{v}^{2}}, \lambda=\frac{\sigma_{u}}{\sigma_{v}}, \mathrm{f}($.$) é a$ função densidade da normal reduzida e $\mathrm{F}($.) é a função distribuição da normal reduzida.

Jondrow, Lovell, Materov e Schmidt (1982) mostraram que o rácio da variação das variáveis $\nu$ e $v$, seja $\lambda$, pode ser tomado como uma medida da ineficiência relativa de um banco:

$$
\lambda=\frac{\sigma_{u}}{\sigma_{v}}
$$

A estimação da ineficiência produtiva de um banco, dada pelo parâmetro $\lambda$, pode ser feita de forma direta, mediante a maximização da função de log-verossimilhança $(\ln \phi)$, decorrente de $\mathrm{g}(\varepsilon)$ - estimação pelo método da máxima verossimilhança:

$\ln \phi=\frac{N}{2} \ln \frac{2}{\pi}-N \ln \sigma+\sum_{i=1}^{N} \ln \left[F\left(\varepsilon_{i} \lambda / \sigma\right)\right]-\frac{1}{2 \sigma^{2}} \sum_{i=1}^{N} \varepsilon_{i}^{2}$

${ }^{17}$ Greene (1993, p. 80-82).

em que $\mathrm{N}$ corresponde ao número de observaçóes, $\lambda=\sigma_{u} / \sigma_{v}, \quad \sigma=\sqrt{\sigma_{u}^{2}+\sigma_{v}^{2}}$, $\varepsilon_{i}=u_{i}+v_{i}$, e $\mathrm{F}($.) é a função distribuição da normal reduzida.
Os resíduos estimados $\varepsilon_{i}$ podem ser decompostos nas suas parcelas, por intermédio do método apresentado por Jondrow, Lovell, Materov e Schmidt (1982), segundo o qual a esperança matemática da distribuição condicionada para o modelo seminormal é dada por:

$E\left(u_{i} \mid \varepsilon_{i}\right)=\frac{\sigma_{u} \sigma_{v}}{\sigma}\left[\frac{f\left(\varepsilon_{i} \lambda / \sigma\right)}{F\left(\varepsilon_{i} \lambda / \sigma\right)}+\frac{\varepsilon_{i} \lambda}{\sigma}\right]$ (8)

$E\left(u_{i} \mid \varepsilon_{i}\right)$ é um estimador cêntrico, mas náo consistente do parâmetro $u_{i}$ , porque, independentemente de $\mathrm{N}$, a variância do estimador é positiva. ${ }^{17}$

A eficiência produtiva global (eficiência-X), definida como o rácio entre o mínimo e o custo atual, é dada por: ${ }^{18}$

$E P-X=\frac{C T\left(\ln q_{i}, \ln w_{i}\right)}{C T_{i}}=e^{-u_{i}-v_{i}}$

onde $q_{i}$ e $w_{i}$ são vetores incluindo os logaritmos dos outputs e os preços dos inputs do i-ésimo produtor. Ignorando o termo de perturbação estocástico $v_{i}$ , o indicador da eficiência produtiva global obter-se-á por:

$$
\mathrm{EP}-\mathrm{X}=e^{-u}
$$


A sensibilidade dos resultados estimados para a ineficiência aos diferentes modelos é comumente aceita. ${ }^{19}$ No entanto, a ordenação dos produtores pela sua ineficiência individual (dos produtores ou por classes) não parece ser sensivelmente afetada pelo modelo adotado. De fato, parece haver correlação muito eleva$\mathrm{da}$ entre os resultados obtidos, assumindo diferentes distribuiçóes para $u,{ }^{20}$ o que permite, nessas circunstâncias, optar por uma distribuição simples para $u$ (como a seminormal ou a exponencial) em vez de distribuiçōes mais complexas.

Nesse sentido, Altunbas, Liu, Molyneux e Seth (2000) e Altunbas, Gardener, Molyneux e Moore (2001) recorrem a uma funçâo Fourier, com dados em painel ${ }^{21}$ (efeitos aleatórios), para estimar a eficiência nos mercados bancários japonês (estudo de 2000) e europeu (estudo de 2001). Do ponto de vista das estimações empíricas, a adoção de diferentes funções densidade de probabilidade para o termo $u_{i}$, como seminormal, normal truncada, exponencial ou gama interfere com os resultados individuais das estimações, como vimos. O recurso a dados em painel diminui esses problemas, dado que permite a estimação das componentes cross-section e temporais de $\varepsilon$ (Schmidt; Sickles, 1984).22

\section{Aplicação empírica: o caso português}

\section{1_O modelo}

A forma funcional Fourier é, como apresentada anteriormente, uma forma mista ou semiparamétrica construída a partir de uma função do tipo Translog, recorrendo a um número limitado de termos trigonométricos, possibilitando melhor ajustamento da funçáo custo. No nosso caso, incorporamos somente os cossenos e os senos de primeira ordem ao ajustamento da função Translog original - expressão (1).

Quando da definição de empresa bancária, adotou-se a abordagem pela intermediação considerando a totalidade dos custos como variável explicada pelo modelo. Ou seja, além dos custos operacionais, são incluídos os custos financeiros.

\author{
truncada e a seminormal). \\ ${ }^{21}$ Os dados cross-section \\ permitem a estimação da \\ eficiência do conjunto dos \\ produtores (conjunto dos \\ bancos), enquanto os dados \\ em painel proporcionam a \\ análise da eficiência de cada \\ banco ao longo de uma \\ sequência temporal. \\ ${ }^{22}$ Citados por Mendes e \\ Rebelo (1999, p. 515).
}


A definição dos inputs apresenta algumas dificuldades.

Quanto ao fator de produção trabalho, é relativamente pacífico que o cômputo do custo unitário é feito através da relação entre a totalidade das despesas com o pessoal e o número de efetivos (dada a inexistência de informação quanto ao número de horas de trabalho).

No referente ao fator capital financeiro, vamos defini-lo como o conjunto dos saldos das contas de depósitos, seguindo trabalhos anteriores. ${ }^{23} \mathrm{~A}$ totalidade dos depósitos pode ser segmentada em depósitos à ordem, a prazo e de poupança e acrescentada de outros recursos equiparados (débitos representados por títulos). $\mathrm{O}$ preço unitário do capital financeiro será resultante do quociente entre juros e custos equiparados e os depósitos totais e débitos representados por títulos.

Quanto ao capital físico, a sua definição é objeto de algumas leituras não coincidentes. ${ }^{24}$

${ }^{23}$ Mendes e Rebelo (1997).

${ }^{24}$ Bernard (1977) e

Sassenou (1992).

${ }^{25}$ Para o caso português, conferir Mendes (1991, 1994).

${ }^{26}$ Pinho (1994).

${ }^{27}$ Produto bancário $=($ juros e proveitos equiparados juros e custos equiparados)
Estudos sobre a banca ${ }^{25}$ sugerem para a definição do custo unitário do capital físico a relação entre o custo do input (amortizaçóes e outros custos) e o ativo total. Outros autores vão no sentido de aproximar o custo unitário do capital físico por meio da soma da taxa de depreciação do imobilizado e do custo de oportunidade do seu financiamento (dada pela taxa média no Mercado Monetário Interbancário), ou seja, socorrendo-se de medidas extracontabilísticas. ${ }^{26}$

No referente à banca, o produto bancário ${ }^{27}$ é utilizado para remunerar o conjunto dos fatores de produção: o capital físico, o capital financeiro e a mão de obra. Daí que alguns autores ${ }^{28}$ proponham como preço unitário do capital físico a relaçáo entre o custo do capital físico, dada a inexistência de informação estatística acerca do preço por metro quadrado de cada estabelecimento (amortizaçóes adicionadas dos encargos e aluguéis decorrentes de operações de leasing e similares), e o stock do fator (somatório das imobilizaçóes líquidas, dos stocks de capital amortizado e das operações de leasing e similares). É essa a solução que se propóe no presente trabalho.

Quanto ao produto bancário, já ficaram expressas as implicações da adoção da abordagem intermediação. Grande parte dos estudos ${ }^{29}$ assume como output bancário a totalidade das aplicaçóes cre- 
ditícias, das aplicações noutras instituições de crédito e das aplicações em títulos.

$\mathrm{Na}$ especificação Fourier, vamos considerar as seguintes variáveis:

$C T \equiv$ Custo Total, incluindo custos operacionais, custos financeiros e as amortizaçóes do exercício.

Produtos:

$\mathrm{CC} \equiv$ Crédito sobre Clientes;

$\mathrm{CIC} \equiv$ Créditos sobre Instituições de Crédito; e

APT $\equiv$ Aplicações em Títulos.

Fatores produtivos e respectivos preços unitários:

1_ Trabalho:

$X_{1} \equiv$ Número de trabalhadores;

$W_{1} \equiv$ preço de $X_{1}$, definido

como a relação entre despesas

com pessoal e número de

trabalhadores.

2_ Capital financeiro:

$X_{2} \equiv$ Saldo das contas

de depósitos e débitos

representados por títulos.

$W_{2} \equiv$ Preço de $X_{2}$, dado pelo

quociente entre juros e custos

equiparados e os depósitos

totais e débitos representados

por títulos.

3_ Capital físico:

$X_{2} \equiv$ Saldo das imobilizaçóes líquidas e dos stocks de capital amortizado.
$W_{3} \equiv$ Preço de $X_{2}$, dado pelo

quociente entre as amortizaçóes

do exercício do fator e as

imobilizações brutas.

4_Outros inputs:

$X_{4} \equiv$ Ativo Líquido;

$W_{4} \equiv$ preço unitário de outros

inputs, definido como a soma

de Prejuízos de Operaçóes

Financeiras, Comissóes e

Outros Custos de Exploração

ponderada pelo Ativo Líquido.

Além dessas variáveis, vão ser considerados os "shares" para cada um dos inputs.

São acrescentadas as variáveis seno (siz) e cosseno (csz) de primeira ordem, de acordo com a lógica da especificação tipo Fourier.

\section{2_A amostra}

As estimaçóes vão ter por base uma amostra de 22 bancos a operar em Portugal, entre 31 de dezembro de 1995 e 31 de dezembro de 2001, correspondendo a um total de 127 observaçóes de registros anuais, após eliminação das observaçôes para as quais não havia dados disponíveis (bancos sujeitos a fusão ou aquisição), tendo o número de observaçóes inicial de 154 observaçóes anuais (22 bancos durante sete anos) sido reduzido. Os dados referem-se à atividade não consolidada dos bancos. 
${ }^{30}$ Cálculo com base nos dados consolidados do Boletim Informativo da Associação Portuguesa de Bancos, de junho de 2002.
Os bancos incluídos na amostra são: Banco BPI (BPI), Banco Borges \& Irmão (BBI), Banco Fonsecas \& Burnay (BFB) e Banco de Fomento e Exterior (BFE) - Grupo BPI; Banco Comercial dos Açores (BCA) e BANIF - Grupo BANIF; Banco Santander (Portugal) (BSP), Banco Totta e Açores (BTA) e Crédito Predial Português (CPP) - Grupo Totta/ Santander; Banco Internacional de Crédito (BIC) e Banco Espírito Santo (BES) Grupo BES; Banco Comercial Português (BCP), Banco Pinto \& Sotto Mayor (BP$\mathrm{SM})$, Banco Português do Atlântico (BPA), Banco Mello (BMello) e Expresso Atlântico (BEXA) - Grupo Millennium BCP; Caixa Geral de Depósitos (CGD) e Banco Nacional Ultramarino (BNU) - Grupo CGD; Barclays Bank (BARC), Banque National de Paris (BNP), Deutsche Bank (DB) e Montepio Geral (MG). As instituições incluídas na amostra representavam 87,6\% da totalidade do Ativo Líquido e $81,1 \%$ da totalidade das Aplicações Creditícias das instituiçóes de crédito que operavam em Portugal, em 31 de dezembro de $2001 .{ }^{30}$

A franja mais importante dos custos totais corresponde aos custos financeiros que, em média e para o período de 1995 a 2001, eram associados a mais de $84 \%$ da totalidade dos custos. O produto é repartido, em média e para o perío- do amostral, entre Créditos sobre Clientes (quase 7), Créditos sobre Instituições de Crédito (29,9\%) e Aplicações em Títulos (16,5\%). O desvio padrão apresenta-se muito elevado ao nível das produçóes, do Ativo Total e dos próprios custos, evidenciando que a amostra comporta instituiçôes de dimensão diversa.

\section{3_Estimação da fronteira estocástica com dados em painel}

Para o estudo econométrico da banca portuguesa, utiliza-se o modelo fronteira estocástica de custo (stochastic frontier model). Considere-se a seguinte especificação:

$\operatorname{Ln} C_{i t}=\alpha+\beta^{\prime} X_{i t}+\left(v_{i t}+u_{i}\right)$

$u_{i}=\left|U_{i}\right| ; \quad U_{i} \approx N\left(0, \sigma_{u}^{2}\right) ;$

$v_{i t} \approx N\left(0, \sigma_{v}^{2}\right)$;

$\operatorname{Var}\left(u_{i}\right)=\left(1-\frac{2}{\pi}\right) \sigma_{u}^{2}$

O termo de perturbação é dado por $\varepsilon_{i t}=v_{i t}+u_{i}$, representado o termo de perturbação $u_{i}$ o efeito específico da ineficiência de cada banco. A fronteira estocástica é dada por $\alpha+\beta^{\prime} X_{i t}+v_{i t}$ (o qualificativo estocástico é justificado pelo termo aleatório $v_{i t}$ na fronteira de eficiência de custo). Esse modelo terá em consideração ainda os efeitos aleatórios, com 
estrutura de dados em painel. Note-se que, sendo $u_{i}$ uma variável aleatória seminormal positiva, obtida a partir da normal $U_{i}$, com média nula e variância $\sigma_{u}^{2}$, a sua variância é dada por $\operatorname{Var}(u)=\left(1-\frac{2}{\pi}\right) \sigma_{u}^{2}$.

Nesse modelo, são relevantes a ineficiência para cada banco, conforme definido por Jondrow et al. (1982). $E\left(u_{i} \mid \varepsilon_{i t}\right)$, e a proporção da variância em razão da ineficiência:

$$
\frac{\operatorname{Var}(u)}{\operatorname{Var}(\varepsilon)}=\frac{\operatorname{Var}(u)}{\operatorname{Var}(u)+\operatorname{Var}(v)}
$$

O método de estimação utilizado é o da máxima verossimilhança (Greene, 1995, presente no software LIMDEP - versões 7 e 8), que, sendo um método não linear, permite ganhos de eficiência (Greene, 2003; Arellano, 2003).

\section{4_Resultados de estimação ${ }^{31}$}

Os resultados da estimação para a especificação Fourier encontram-se na Tabela 1 a seguir. Uma das razóes deste trabalho é justamente o de identificar para cada unidade bancária as respectivas taxas de ineficiência, pelo que se coloca outra questão lógica e estatística que é a de saber se se deve usar a função Translog ou a Fourier (esta acrescenta à Translog alguns termos trigonométricos) de suporte a esses cálculos.
Em relação às variáveis explicativas, deve notar-se que algumas delas são estatisticamente significativas ao nível individual, mas o fato de algumas outras não o serem não implica a invalidação da função, sob pena de se ficar com variáveis justificadas apenas empiricamente, mas a que faltaria a compreensão lógica própria da função Tanslog - adaptada à produção multiproduto e à substituição de fatores e produções. A função deve, pois, manter-se com as suas principais características, ou seja, trata-se de um imperativo teórico. Já a passagem da função Translog à função Fourier só se justificará se as variáveis adicionadas (em senos e cossenos) forem suportadas empiricamente - lembremos que a funçáo Fourier, ao acrescentar os termos trigonométricos, pretende melhorar, em relação à função Translog, o ajustamento local.

No nosso estudo, a especificação da função Fourier, para determinação da fronteira estocástica, revela que, das seis variáveis acrescentadas em relação à função Translog, as variáveis que incorporam o crédito a clientes (CSZCC e SIZCC) são individualmente significativas (ao nível de significância de $10 \%$ ), concluindo-se que existe diferença entre as duas especificaçóes no ajustamento dos custos.

A significância estatística das variáveis seno e cosseno referidas, típicas da função

\footnotetext{
${ }^{31}$ A especificação da função Fourier exige o cálculo simultâneo das condiçôes dos "shares". No entanto, não foram consideradas essas restrições no processo de estimação.
} 
Tabela 1_Resultados de estimação da fronteira estocástica para a especificação Fourier

\begin{tabular}{|c|c|c|c|c|}
\hline Variáveis & Coeficientes & Desvio padrão & Coef./Desvio padrão & $\mid \mathrm{P}[|\mathrm{T}|>\mathrm{t}]$ \\
\hline Constante & 31,117 & 15,130 & 2,057 & 0,040 \\
\hline LN2APT & 0,030 & 0,029 & 1,003 & 0,316 \\
\hline LN2CC & 0,468 & 0,172 & 2,711 & 0,007 \\
\hline LN2CIC & 0,092 & 0,093 & 0,988 & 0,323 \\
\hline LNAPT & 0,486 & 0,293 & 1,660 & 0,097 \\
\hline LNCC & $-3,143$ & 2,045 & $-1,537$ & 0,124 \\
\hline LNCCAPT & $-0,079$ & 0,028 & $-2,827$ & 0,005 \\
\hline LNCCCIC & $-0,073$ & 0,024 & $-3,080$ & 0,002 \\
\hline LNCIC & $-0,972$ & 1,232 & $-0,789$ & 0,430 \\
\hline LNCICAPT & 0,032 & 0,024 & 1,362 & 0,173 \\
\hline LN2W1 & $-0,161$ & 0,335 & $-0,482$ & 0,630 \\
\hline LN2W2 & 0,276 & 0,156 & 1,769 & 0,077 \\
\hline LN2W3 & $-0,220$ & 0,157 & $-1,400$ & 0,162 \\
\hline LN2W4 & 0,210 & 0,013 & 15,701 & 0,000 \\
\hline LNW1 & 0,153 & 1,023 & 0,150 & 0,881 \\
\hline LNW1W2 & 0,057 & 0,198 & 0,289 & 0,773 \\
\hline LNW1W3 & 0,008 & 0,210 & 0,038 & 0,970 \\
\hline LNW1W4 & 0,009 & 0,069 & 0,128 & 0,899 \\
\hline LNW2 & 0,812 & 0,915 & 0,888 & 0,375 \\
\hline LNW2W3 & 0,095 & 0,093 & 1,022 & 0,307 \\
\hline LNW2W4 & $-0,151$ & 0,062 & $-2,453$ & 0,014 \\
\hline LNW3 & $-0,777$ & 0,736 & $-1,056$ & 0,291 \\
\hline LNW3W4 & 0,005 & 0,067 & 0,073 & 0,942 \\
\hline LNW4 & 0,840 & 0,275 & 3,057 & 0,002 \\
\hline LNAPTW1 & $-0,010$ & 0,058 & $-0,179$ & 0,858 \\
\hline LNAPTW2 & 0,331 & 0,047 & 0,709 & 0,478 \\
\hline LNAPTW3 & $-0,013$ & 0,042 & $-0,300$ & 0,764 \\
\hline LNAPTW4 & $-0,003$ & 0,015 & $-0,175$ & 0,861 \\
\hline LNCCW1 & $-0,122$ & 0,123 & $-0,990$ & 0,322 \\
\hline LNCCW2 & 0,056 & 0,055 & 1,015 & 0,310 \\
\hline LNCCW3 & 0,070 & 0,091 & 0,771 & 0,441 \\
\hline LNCCW4 & $-0,005$ & 0,021 & $-0,234$ & 0,815 \\
\hline LNCICW1 & 0,161 & 0,093 & 1,734 & 0,083 \\
\hline LNCICW2 & $-0,082$ & 0,047 & $-1,736$ & 0,083 \\
\hline LNCICW3 & $-0,026$ & 0,064 & $-0,396$ & 0,692 \\
\hline LNCICW4 & $-0,006$ & 0,022 & $-0,253$ & 0,800 \\
\hline CSZAPT & 0,083 & 0,345 & 0,240 & 0,811 \\
\hline CSZCC & $-0,699$ & 0,368 & $-1,898$ & 0,058 \\
\hline CSZCIC & $-0,010$ & 0,162 & $-0,062$ & 0,951 \\
\hline SIZAPT & 0,025 & 0,254 & 0,100 & 0,921 \\
\hline SIZCC & $-0,766$ & 0,402 & $-1,905$ & 0,057 \\
\hline ȘIZC̣IC. & $-0,092$. & 0,086 . & $-1,062$. & 0,288 . \\
\hline
\end{tabular}

$$
\begin{gathered}
\sigma_{u}^{2}=0,00282 \\
\sigma_{v}^{2}=0,00166
\end{gathered}
$$

$\operatorname{Var}(u)=0,001025$
$\frac{\operatorname{Var}(u)}{\sigma_{v}^{2}}=0,617$
$\sigma=\sqrt{\sigma_{u}^{2}+\sigma_{v}^{2}}=0,0669$
$\lambda=\frac{\sigma_{u}}{\sigma_{v}}=1,303$

Fourier, implica que o modelo Fourier de explicação da ineficiência é significativamente preferível ao modelo Translog, o que permite concluir pela superioridade da função Fourier no nosso estudo e justificar a sua recomendação para outros estudos de eficiência bancária.

Das duas partes que contribuem para a variância total, a relativa à ineficiência é apenas 0,617 da outra fonte.

A estimação da fronteira estocástica com a especificação Fourier sugere a possibilidade de poupança de custos totais, a não haver ineficiência, de 4,2\%, em termos médios. 
Tabela 2_Medidas estatísticas descritivas da ineficiência especificação Fourier

\begin{tabular}{r|r|r|r|r} 
& Média & Máximo & Mínimo & Desvio padrão \\
\hline Taxa de Ineficiência & 4,2 & 13,4 & 1,0 & 2,1
\end{tabular}

Analisando-se a eficiência por banco, obtemos os resultados expressos na Tabela 3 , em que os valores médios vão de $2,88 \%$ a $7,61 \%$, mostrando que as diferentes entidades bancárias têm capacidade de gestão da eficiência produtiva diversificada e que, para as amplitudes verificadas, podem resultar consequências derivadas sobre a capacidade competitiva das marcas em concorrência, bem como sobre a rentabilidade bancária e a respectiva cotação em bolsa, pelo que a gestão deve prestar maior atenção às questóes da eficiência. De notar que a Tabela 3 mostra já os valores da ineficiência em taxa de variaçáo re- lativa (em \%), correspondentes ao índice $\exp (-\mathrm{u})$, anteriormente apresentado.

O Gráfico 1 pretende mostrar a variabilidade ao longo dos sete anos da ineficiência produtiva para cada marca bancária. Note-se que o valor mínimo calculado para a taxa de ineficiência é de $1 \%$, e o valor máximo é de $13,4 \%$, o que reforça a importância da qualidade de gestão dos recursos produtivos e a devida atenção às flutuaçóes anuais dos preços desses recursos (trabalho, capital físico e capital financeiro) e também dos outputs - exigindo-se rápida adaptação sob pena de maior ineficiência e, em consequência, mais fraca rentabilidade e competitividade.

Tabela 3_Ineficiência por banco (média do período) - especificação Fourier

\begin{tabular}{l|r|r|r}
\hline Banco & Taxa de Ineficiência (média) & Banco & Taxa de Ineficiência (média) \\
\hline CGD & 4,66 & CPP & 3,46 \\
\hline BNU & 3,34 & BSP & 4,31 \\
\hline BCP & 7,61 & BANIF & 5,11 \\
\hline BPA & 3,47 & BCA & 4,57 \\
\hline BEXA & 3,62 & BFE & 3,55 \\
\hline BPSM & 5,85 & BPI & 3,72 \\
\hline BMELLO & 5,38 & BBI & 3,40 \\
\hline BES & 3,72 & DB & 3,64 \\
\hline BIC & 2,88 & BFB & 3,07 \\
\hline BTA & 3,57 & MG & 4,10 \\
\hline BARC & 4,39 & BNP & 3,95
\end{tabular}


Gráfico 1_Taxas de ineficiência por banco - especificação Fourier

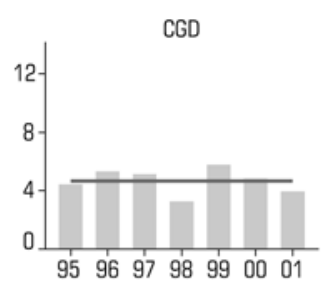

BPSM

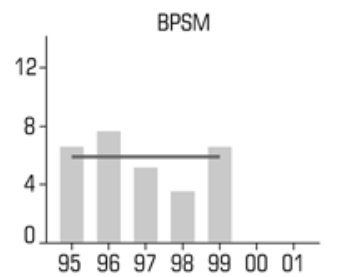

CPP

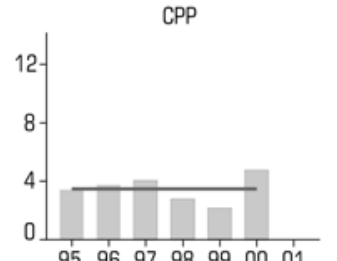

BPI

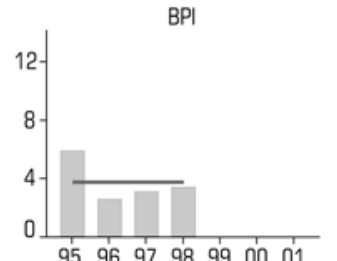

BARC

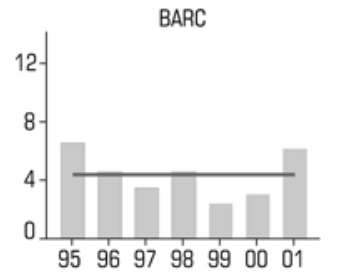

BNU

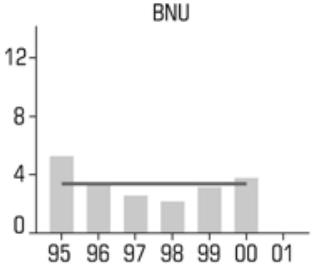

BMELLO

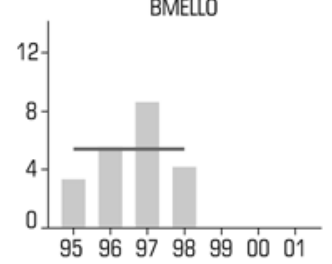

BSP

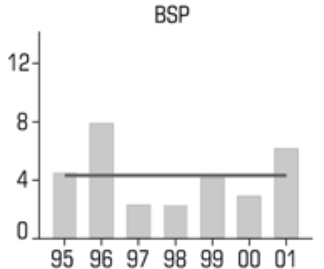

BBI

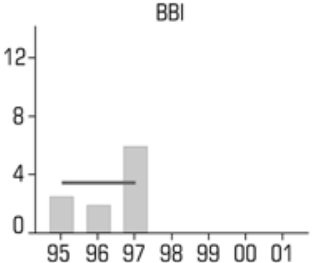

BNP

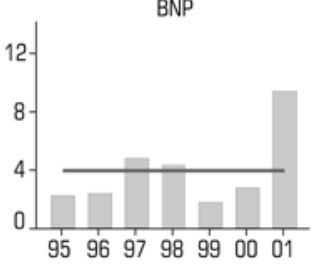

BCP

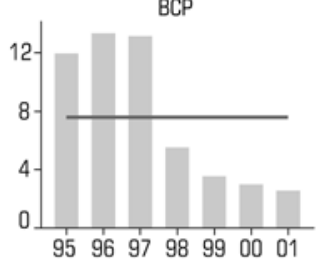

BES

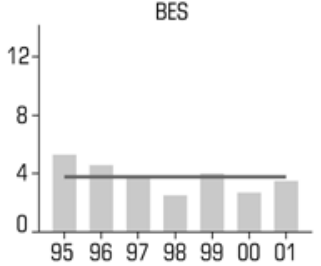

BANIF

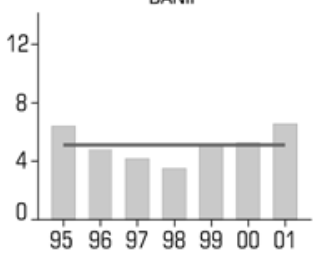

BFB

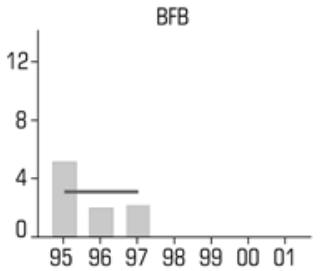

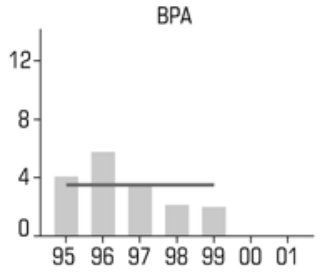

BIC

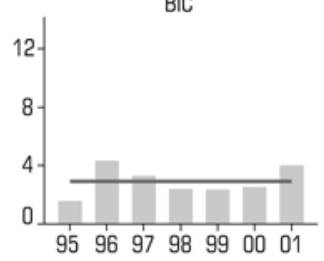

BCA

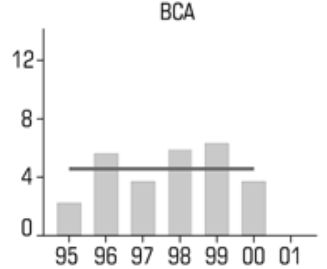

MG

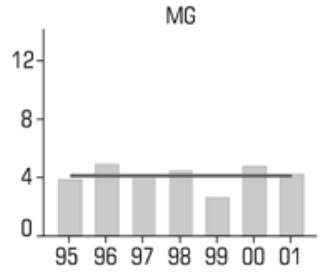

BEXA

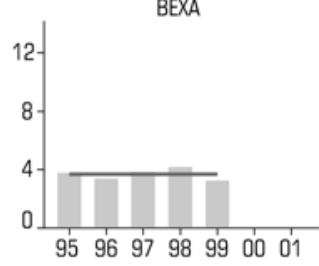

BTA

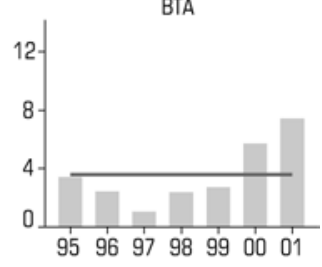

BFE

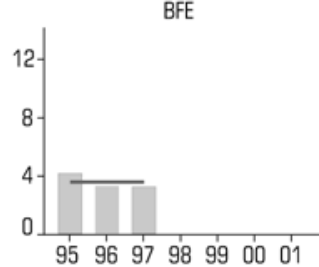

DB

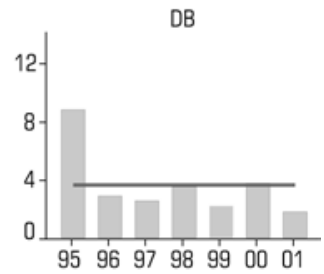

Taxa de ineficiência anuais (\%) — Taxa média de ineficiência (\%) 
No teste de a hipótese das taxas médias de ineficiência serem iguais entre os bancos (contra a hipótese alternativa de serem diferentes), conclui-se pela existência de diferenças estatísticas significativas (rejeita-se $H_{0}$ a 5\%, estatística F de 1,792). O teste estatístico está na mesma linha conclusiva das taxas de ineficiência calculadas, podendo resumir-se que, apesar de não haver ineficiência muito elevada, existem unidades bancárias bastante mais eficientes que outras, o que poderá levar a maior desequilíbrio nos mercados bancários de crédito e depósitos, com consequências agravadas sobre a rentabilidade e o valor bolsista das unidades bancárias, caso a situação não seja revertida por meio da promoção de medidas de eficiência adequadas.

\section{5_Conclusão}

Neste estudo percorreu-se a literatura bancária em torno da problemática da eficiência produtiva. As condiçóes decorrentes da teoria da dualidade, entre produção e custos, permitiram a opção pela abordagem centrada nos custos. A não necessidade de especificar, previamente, as condiçóes tecnológicas de produção (ultrapassando o obstáculo da especificação, assim como a perda de generalidade), por um lado, e a maior fiabilidade e facilidade no que con- cerne à recolha dos dados necessários ao processo de estimação, por outro, são premissas justificadoras dos desenvolvimentos via custos em detrimento da opçáo baseada na produçáo.

Dos debates teóricos fundamentais acerca da definição econômica de empresa bancária, a abordagem intermediação foi a adotada, quer por razóes de natureza econômica (peso que os custos financeiros assumem na totalidade dos custos bancários), quer de ordem empírica (inexistência de informação para a adoção da abordagem produção para o caso português). Decorrente do caráter multiproduto da atividade bancária, conclui-se pela necessidade do recurso a formas funcionais flexíveis de funções custo, tendo-se eleita a função Fourier, por causa da sua capacidade de adaptabilidade aos dados (por via da introdução de senos e cossenos).

Para o cômputo da ineficiência sobre os custos bancários portugueses, foi adotada a forma funcional Fourier - tendo por suporte dados em painel, para o período de 1995 a 2001, comportando 22 instituiçốes bancárias.

A estimação da ineficiência, recorrendo à função Fourier (dados em painel, efeitos aleatórios), permite obter taxas de ineficiência média, para o período amostral, de 4,2\%. Parece concluir-se ser 
possível que as instituiçóes portuguesas possam reduzir os seus custos totais, diminuindo a ineficiência-X.

O modelo apresentado poderá ser objeto de melhoramentos em futuras investigaçóes, nomeadamente: a estimação, com uma amostra mais alargada temporalmente, dos efeitos das fusóes e aquisiçóes; a consideração, após processo de concentração, da internacionalização previsível dos grupos portugueses; a ponderação de modelos alternativos e o aperfeiçoamento econométrico ao nível da heterogeneidade e da evolução temporal presente nos dados. Esses pontos poderão ser uma via de obtençáo de novos resultados, possibilitando melhor caracterização da banca, mais próxima da realidade e dos desafios que o mercado bancário apresenta. 


\section{Referências bibliográficas}

\begin{abstract}
AIGNER; Dennis; LOVELL, Knox C. A.; SCHMIDT, Peter. Formulation and estimation of stochastic frontier production function models. Journal of Econometrics, 6, p. 21-37, 1977.
\end{abstract}

ALTUNBAS, Y.; CHAKRAVARTY, S. P. Frontier cost functions and bank efficiency. Economics Letters, 72, p. 233-240, 2001.

ALTUNBAS, Y.; GARDENER, E. P. M.; MOLYNEUX, P.; MOORE, B.

Efficiency in European banking.

European Economic Review, 45, p. 1931-1955, 2001.

ALTUNBAS, Yener; LIU, MingHau; MOLYNEUX, Philip; SETH, Rama. Efficiency and risk in Japonese Banking. Journal of Banking \& Finance, 24, p. 1605 1628, 2000.

AREllano, Manuel. Panel Data Econometrics. Oxford University Press, 2003.

BARROS, Pedro Pita; SOARES DE PINHO, Paulo. Estudos sobre o sistema bancário português. Lisboa: Edição Banco Mello, 1995.

BERGER, A. N. The profit-structure relationship in banking - Tests of market-power and efficientstructure hypothesis. Journal of Money, Credit and Banking, v. 27, n. 2, p. 404-431, 1995.
BERGER, A.; HANWECK, G.; HUMPHREY, D. Competitive viability in banking: Scale, scope and product mix economies. Journal of Monetary Economics, p. 501-520, 1987.

BERGER, A.; HUNTER, W. C.; TIMME, S. G. The efficiency of financial institutions: A review and preview of research past, present and future. Journal of Banking and Finance, v. 17, n. 2-3, p. 221-50, 1993.

BERGER, Allen N.; LEUSNER, John H.; MINGO, John J. The efficiency of bank branches. Journal of Monetary Economics, 40, p. 141-162, 1997.

BERNARD, A. Le coût du capital productif: Une ou plusieurs mesures? l'INSEE, no 28, 1977. Annales..

BINSWANGER, H. P. A cost function approach to the measurement of elasticities of factor demand and elasticities of substitution. American Journal of Agricultural Economies, 56, p. 377-386, 1974.
CANHOTO, Ana (1999).

Efficiency and competition in portuguese banking: An empirical investigation. 1999. f. Tese (Doutorado em Economia) Universidade Católica Portuguesa, Lisboa, 1999.

CARBOT, S.; GARDENER, E. P. M.; WILLIAMS, J. A note on technical change in banking: The case of European saving Banks. Applied Economics, 35, p. 705-719, 2003.

D'OLIVEIRA, Eduardo L. The theory of banking: A critical survey of the literature. The Open University, July, p. 27-31, 1984.

DIETSCH, Michel; LOZANO-

VIVAS, Ana. How the environment determines banking efficiency: A comparison between French and Spanish industries. Journal of Banking \& Finance, 24, p. $985-1004,2000$

\section{DIEWERT, W. E. The} measurement of productivity. Bulletin of Economic Research, 44, p. 163-98, 1992.

FARE, R.; PRIMONT, D. Multi-output production and duality: Theory and applications. Kluwer Academic Publishers, Boston, 1997.
GALLANT, A. R. On the bias in flexible functional forms and essentially unbiased form: The Fourier flexible form. Journal of Econometrics, 15, p. 211-245, 1981.

GALLANT, A. R. Unbiased determination of production technologies. Journal of Econometrics, 20, p. 285-324, 1982.

GREENE, W. M. A gammadistributed stochastic frontier model. Journal of Econometrics, 46, p. $141-163,1990$.

GREENE, W. M. The econometric approach to efficiency analysis. In: FRIED, H. O.; LOVELL, C. A.; SCHMIDT, P. (Eds.). The measurement of productive efficiency: Techniques and applications. Oxford University Press, 1993.

GREENE, W. M. Econometric analysis. Prentice Hall, $5^{\text {th }}$ edit, 2003.

GREENE, W. M. LIMDEPversion 7-User's Manual. Econometric Software, Inc., 1995. HUMPHREY, David B. Cost and scale economies in banking intermediation. Ed. John Wiley and Sons, 1985. 
HUMPHREY, David B.; VALE, Bent. Scale economies, bank mergers, and electronic payments: A spline function approach. Journal of Banking \& Finance (article in press), 2003.

JEHLE, Geoffrey A. Advanced Microeconomic Theory. London: Prentice-Hall International, 1991.

JONDROW, J.; LOVELL, C. A.; MATEROV, I. S.; SCHMIDT,

P. On estimation of thecnical inefficiency in the stochastic frontier production model. Journal of Econometrics, 19, p. 233-38, 1982.

JORGENSON, D. W. Econometric methods for modelling producer beahaviour. Handbook of

Econometrics, p. 1841-1915, 1986.

KOLARI, J.; ZARDHOOHI, A.

Bank cost, struture and performance.

Lexington Books, 1987.

KUMBHAKAR, Subal C.; LOVELL, C. A. Knox, Stochastic Frontier Analysis. Cambridge University Press, 2003.

LAU, L. J. Functional forms in Econometric Model Building. Handbook of Econometrics, p. 1515-1552, 1986.

LEIBENSTEIN, H. Allocative efficiency vs X-efficiency. American Economic Review 2 56, p. 392-15, 1966.

MENDES, Victor. Scale and scope economies in portuguese commercial banking: The years 1965-88. Economia, Separata do vol. XV, n. 3, out. 1991.
MENDES, Victor. Eficiência produtiva no sector bancário: Uma aplicação do método DEA aos anos 1990-92. Trabalhos em Curso, 42, Faculdade de Economia da Universidade do Porto, 1994.

MENDES, Victor; REBELO, João. Relaçóes custo-produção e eficiência produtiva no sistema integrado de crédito agrícola mútuo no período 1990-1995. Estudos de Economia, v. XVI-XVII, n. 2, p. 117-35, 1997.

MENDES, Victor; REBELO, João. Productive efficiency, technological change and productivity in Portuguese banking. Applied Financial Economics, 9, p. 513-521, 1999.

MITCHELL, Karlyn; ONVURAL, Nur M. Economies of scale and scope at large commercial banks: Evidence from the fourrier flexible form. Journal of Money, Credit and Banking, 28, p. 178-199, 1996.

MOLYNEUX, P.; ALTUNBAS, Y.; GARDENER, E. Efficiency in European banking. Ed. John Willey \& Sons, Lda., England, 1996.

PINHO, Paulo. Essays on banking. Doctoral Dissertation, City University Business School, 1994.

RESTI, Andrea. Evaluating the cost-efficiency of the Italian banking system: What can be learned from the joint application of parametric and non-parametric techniques. Journal of Banking \& Finance, 21, p. 221-50, 1997.
RESTI, Andrea. Efficiency measurement for multi-product industries: A comparison of classic and recent techniques based on simulated data. European Journal of Operational Research, 121, p. 559-578, 2000.

SASSENOU, Mohamed.

Economies des coûts dans les banques et les caisses d'épargne, impact de la taille et de la variété de produits. Revue Économique, v. 43, n. 2, p. 277-50, 1992.

SCHMIDT, P.; SICKLES, R.C.. Production Frontiers and Panel Data, Journal of Business \& Economic Statistics, 2, n. ${ }^{\circ}$ 4, p.367-374

SEALEY, C. W.; LINDLEY, J. Inputs, outputs and a theory of production and cost at depository financial institutions. Journal of Finance, 34, p. 1251-65, 1977.

TOLSTOV, G. P. Fourier series.

London: Prentice-Hall, 1962.

\section{Outras publicações:}

Associação Portuguesa de Bancos. Boletim Informativo (vários números).

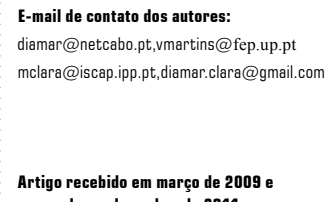


